\title{
Hierarchie oder Verantwortung durch klinisch- psychologische Diagnostik in der Psychotherapie?
}

\section{Überlegungen zu einer integrierten Sichtweise im Rahmen der Psychotherapie}

\author{
Angéla Szalontainé Krasznai
}

Psychotherapie-Wissenschaft 8 (1) 35-44 2018

www.psychotherapie-wissenschaft.info

CC BY-NC-ND

DOI: $10.30820 / 8242.07$

Zusammenfassung: Wovon hängt die Stellungnahme der PsychotherapeutIn während des psychotherapeutische Prozesses $a b$ ?

Im Psychotherapieprozess erweist sich die Rolle der Diagnostik in mehrfacher Hinsicht als fragwürdig: Einerseits zeigt sich die Diagnostik im klinischen Bereich einem pathologie- und defizitorientierten medizinischen Modell verpflichtet, aus dem heraus die Indikationen zu einer Therapie gestellt werden. Andererseits richtet sich aufgrund der verschiedenen therapeutischen Richtungen die Diagnostik psychischer Störungen auf die linienspezifischen Phänomenbereiche auf die sich das medizinische Modell nur bedingt anwenden lässt. Die Fragestellung kann provokant jedoch auch zukunftsweisend sein. Je nach individuellem Mass an Fachinteresse bzw. entsprechend der Einstellung kann man den Ausführungen folgen.

Mein Beitrag macht den Versuch, einige Aspekte und die sich daraus ergebenden Fragen und Probleme sowie die Vorteile bezüglich der ärztlichen Zusammenarbeit in der klinischen Diagnosestellung zu thematisieren und ihr in der Psychoterapie einen individuellen Platz zu geben durch die «Fachidentität».

Schlüsselwörter: klinisch-psychologische Diagnostik, Diversität, Authentizität, Modelle, Rolle, Psychotherapie, Fachidentität

\section{Grundlegende Definitionen der (klinisch-psychologischen) Diagnostik}

I.1. Definitionen und Abgrenzug: Diagnostik, Klinische Psychologie, Rehabilitationspsychologie, Psychotherapie

Die Wörter «Diagnose» und «Diagnostik» gehen auf das griechische Verb «diagnoskein» zurück. Es bezeichnet unterschiedliche Aspekte eines kognitiven Vorgangs, ist gewissermaßen der Inbegriff vom Erkennen bis zum Beschliessen.

Die psychologische Diagnostik (psychological assessment) ist ein Teilgebiet der Psychologie und zugleich ein bedeutender Teil der Berufstätigkeit der PsychologInnen. R. Jäger und F. Petermann (1999) erstellten eine umfassende Definition für psychologische Diagnostik, welche die Orientierung und eine unglaubliche Komplexität an diagnostischen Entscheidungen betont. Dabei geht es um das systemische Sammeln und Aufbereiten von Informationen mit dem Ziel, Entscheidungen und daraus resultierende Handlungen zu begründen, zu kontrollieren und zu optimieren.

Im weiteren Sinn wird die klinisch-psychologische Diagnostik als Methodenlehre im Dienste der Angewandten Psychologie verstanden. In den Definitionen wird meistens betont, dass die systemische Erfassung relevanter Daten und das daraus erarbeitete diagnostische Urteil (aufgrund der subjektiven Wahrnehmungen oder messbaren Angaben) im Dienste einer Aufgabenstellung aus einem Anwendungsfeld erfolgt und ständig persönliche Veränderungen ermöglichen kann. Psychologische Diagnostik in der klinischen Psychologie bezieht sich nicht nur auf das Erkennen von psychischen Erkrankungen oder Störungen, sondern bezieht die daraus abzuleitenden Fachentscheidungen mit ein, deswegen wird von «Indikationsdiagnostik» gesprochen.

Die psychologische Diagnostik kann bei der Erfassung einer psychischen Störung, in der Rehabilitationspsychologie und im Rahmen von Psychotherapie interpretiert werden (vgl. Linster, 2010). Das betrifft die Startdiagnostik und auch die während des Verlaufs vorkommende weitere Diagnostik bis zur «Schlussdiagnostik». So kann darunter ein «Entwicklungsprozess mit Fachbegleitung» verstanden werden, was die Erweiterung der Definitionsrahmen und weitere Abklärungen der Grunddefinitonen voraussetzt.

Klinische Psychologie ist eine Teildisziplin, die sich mit psychischen Aspekten somatischer Störungen und Krankheiten in der Forschung, Diagnostik und Therapie beschäftigt. Dazu gehören unter anderem Themen wie Äthologie, Bedingungsanalyse, Klassifikation und Diagnostik, Prävention, Psychotherapie und Rehabilitation, Epidemologie, Gesundheistversorgung und Evaluation. Aufbauend auf den wissenschaftlichen Grundlagen der 
Psychologie mit ihren Teildisziplinen, ist es ein Charkteristikum der klinischen Psychologie, dass sie enge Beziehungen zu vielen anderen Wissenschaftsdisziplinen aufweist (Interdisziplinäre Grundorientierung) (vgl. Wittchen \& Hoyer, 2006).

Wenn von dem oben erwähnten Entwicklungsprozess und vom dynamischen Entwicklungsprozess von Diagnostik in der Psychotherapie ausgegangen wird, erweist es sich aus meiner Sicht als notwendig, dazugehörige Begriffe auch zu definieren.

Rehabilitation zielt auf die Beseitigung oder Verringerung der Folgen einer chronischen Erkrankung, einer Behinderung oder eines spezifischen akuten Ereignisses (zum Beispiel eines Unfalls, einer Krise). Sie dient damit der Vorbeugung einer drohenden Behinderung oder der Vermeidung der Verschlimmerung einer bestehenden gesundheitlichen Schädigung (zum Beispiel Chronifizierung). Trotz eventuell bleibender Gesundheitsschäden und ihrer Folgen sollen Menschen mit (beginnenden) chronischen Krankheiten oder Behinderungen in die Lage versetzt werden, möglichst gut mit den alltäglichen Anforderungen zurechtzukommen und ihre Rollenverpflichtungen in Familie, Beruf und Gesellschaft möglichst weitgehend zu erfüllen (vgl. Bengel \& Helmes, 2005) bzw. die individuelle Coping-Strategie auch zu verbessern und die Persönlichkeit zu entwickeln. Die köperlichen und psychischen Verbesserungen und Änderungen sind voneienander nicht zweckmässig zu trennen. Sie wirken nämlich sowohl auf das Individuum als auch das soziale Umfeld und in weiterer Sicht indirekt auf die Gesellschaft. Das kann weitere Auswirkungen und/oder Einflussfaktoren hervorrufen.

In der Rehabilitationspsychologie oder bei den «rehabilitationspsychologische Massnahmen» wird im Rehabilitationsprozess zwischen verschiedenen Aufgaben unterschieden: psychologische Eingangs-, Förder- und Belastungsdiagnostik und Indikationsstellung, psychologische Beratung und Psychotherapie von Patienten und Angehörigen, Patientenschulung und Gesundheitsförderung (vgl. ebd.), Vorbereitung der Reintegration, möglicherweise Wiederherstellung der Arbeitsfähigkeit auf dem Arbeitsmarkt.

Im psychotherapeutischen Rahmen wird die gesetzlich verankerte Massnahme zur Behandlung von Menschen mit psychischen Krankheiten definiert (PsyG, Psychologieberufegesetz in der Schweiz), wobei «Professionalisierung» und "Qualifizierung» durch Ausbildung und Weiterbildung (PsyKo, Psychologieberufekommission in der Schweiz) bzw. (Facharzt) sowie - zur Regelung der Zulassung zur Weiterbildung - die heilsberufliche Tätigkeit und - bei freiberuflicher Tätigkeit - die Arbeit als Vertragspsychotherapeut unter dem Stichwort der «Institutionalisierung» im Rahmen des gesetzlichen Gesundheitssystems (PsyV, Psychologieberufeverordnung in der Schweiz) geregelt werden.

Damit unterliegt die Psyhotherapie den Bestimmungen der gesetzlichen Regelungen. Sie hat sich teilweise gegenüber den Herkunftsdisziplinen Medizin und Psychologie «verselbstständigt» und sich permanent weiterentwickelt.

Psychotherapie wurde und wird üblicherweise von der Medizin und von der Psychologie als Fach reklamiert und «betrieben», daneben existieren auch Beiträge von Personen ausserhalb von Medizin und (akademischer) Psychologie (vgl. hierzu Linster, 2010), somit wird heutzutage als relativ neuer, wichtiger Aspekt die therapeutische Rolle und «die dahinter funktionierende Persönlichkeit» immer mehr erörtert. In der Psychotherapie geht es um einen bewussten und geplanten interaktionellen Prozess (vgl. Strotzka, 1975), auch mit «individueller Präsenz» durch Authentizität und «wahrem Dasein». Gemäss Theodor Itten (2016 bedeutet die Wahrhaftigkeit eine subjektive engagierte symbolische Präsenz in der Psychotherapie. Aus meiner Sicht wäre die Psychotherapie ein Entwicklungsprozess zur Beeinflussung von Verhaltensstörungen und Leidzuständen in einem gemeinsam aufgebauten Konsensus, welcher durch Kommunikation auf beiden «Seiten» (Therapeut, unter Beschwerden leidende Person) auf der menschlichen Ebene eine tragfähige, gegenwärtige und gegenseitige therapeutische Beziehung ermöglicht. Dies kann dazu führen, dass die Prozessbeteiligten sich individuell weiterentwickeln können und dauerhafte Auswirkungen und/oder Nachwirkungen auf diversen Ebenen (individuelle Ebene, familiäre Ebene, soziales Umfeld und Gesellschaft) haben. Der bekannte österreichische Mediziner, Tiefenpsychologe und Psychotherapeut Hans Strotzka definiert sie (die Psychotherapie) als einen bewussten und geplanten interaktionellen Prozess mithilfe psychologischer Mittel zur Beeinflussung von Erlebens- und Verhaltensstörungen sowie Leidenzuständen, die in einem Konsens zwischen Patient, Therapeut und Bezugsperson für behandlungsbedürftig gehalten werden. Dazu zählt Kommunikation, meist verbal, aber auch averbal, in Richtung auf ein definiertes, nach Möglichkeit gemeinsam erarbeitetes Ziel (Symptomminimalisierung und/oder Strukturänderung der Persönlichkeit) mittels lehrbarer Technik auf der Basis einer Theorie des normalen und pathologischen Verhaltens, wofür in der Regel eine tragfähige, emotionale Bindung notwendig ist (vgl. Strotzka, 1975). Die Psychotherapie findet zwangsläufig im Rahmen einer Beziehung statt - dies gilt als ein gemeinsames Wirkprinzip für jede Psychotherapie (vgl. Sedlak \& Gerber, 1976) und bedeutet eine hilfreiche Allianz zwischen Patienten und Therapeuten (vgl. Orlinsky \& Ronnestad, 2005).

Die Wichtigkeit der therapeutischen Beziehung in der Psychotherapie ist unbestrittene Tatsache, was jedoch darunter verstanden werden kann, wird zu einer interessanten Fragestellung: Ein Dilemma sowie eine ziemlich grosse Vielfältigkeit können evoziert werden.

Ein Vorteil und ebenso ein Nachteil bei den Definitionen ist: Die sogenannte «allgemeine Gültigkeit» der methodischen Prinzipien der klinisch-psychologischen Diagnostik und die Diversität geben einen bestimmten Rahmen vor, dieser bestimmt auch die verschiedenen Anwendungsfelder. Der Rahmen engt allerdings die Arbeit in den unterschiedlichen Funktionen, die Prozessmerkmale, Vorgehensweisen und Rollenbedeutungen ein. Was kann das «mit uns» als Therapeut oder Arzt anstellen? Kann die Rollenbedeutung objektiv beurteilt oder eingeschätzt werden? 
Eine Frau sitzt bei mir im Büro. Sie fragt: «Was habe ich?» Und sie seufzt tief. «Warum leide ich schon so lange unter Depressionen? Warum kann ich keine Freude mehr haben? Ich verstehe das nicht.»

Ich antwortete: "Wissen Sie, ich weiss das eben auch nicht. Zwar wurden verschiedene Diagnosen schon gestellt, Sie kennen diese, nicht wabr? Ich kann Ihnen sagen, wie Ihre psychischen Symptome entstanden sind, allerdings kann ich diese noch nicht interpretieren.»

Die Fran schweigt und sagt: "Ich dachte, dass Sie das antworten werden. Geben Sie mir endlich mal Klarheit! Ich will wieder gesundwerden.»

Ich entgegne: "Wissen Sie, die Psychotherapie sieht bei mir so aus: Sie hatten bis jetzt sehr viele positive und sicher auch schwierige Erlebnisse. Heute treffen wir uns und ich nehme an, dass Sie in der Therapie schon einiges erreichen wollen. Stellen Sie sich vor, wie Sie Ibre Ziele erreichen können, kommen Sie und arbeiten Sie mit. Noch weiss ich nicht, wie viele Male Sie kommen müssen. Sehen Sie jede Sitzung als Symbol für Bausteine, mit denen wir eine innere Brücke über Ibr inneres Loch bauen. Sobald Sie über Ibre Brücke gehen können, werden Sie Ihre Stabilität und Ihr Woblfühlen deutlich spüren. Und nach dem Abschluss der Therapie gehen Sie weiter in Ihre Zukunft.»

Die Fran sagt: "AHA. Ah so. Jetzt füblt es sich besser an.»

Ich erkläre weiter: "Wissen Sie, die Diagnose hilft, UNSERE ARBEIT zu klären und Ibren aktuellen Zustand gut, wissenschaftlich zu verstehen, aber das gibt keine konkrete Anweisung für das Finden ibres Weges. Ich begleite Sie, bis Sie Ihre eigene Antwort finden können.»

Wenn ich der Klientin eine konkrete Antwort gebe, brächte ich sie um die Erfahrung, ibre Eigenverantwortung zu übernehmen. Aufgrund meiner Fachidentität vermeide ich bewusst, meine Machtposition aufrecht $z u$ erhalten und die Hilfe suchende Person in ibrem alten Muster zu bestätigen.

Meiner Meinung nach kann die Rollenbedeutung nur durch unsere bzw. individuelle Bewusstheit «objektiviert werden» und kann erst dann, wenn eine Therapie sich ihrem Abschluss nähert, vorsichtig eingeschätzt werden.

\section{I.2. Der Prozess der Diagnosestellung}

Unter dem Diagnosestellungsprozess in der klinischen Psychotherapie kann eine multidimensionale und komplexe Facharbeit begriffen werden. Wenn wir diesen Schritt im Psychotherapieprozess platzieren würden, dann erhielte er in der Regel einen Ausgangsplatz. Durch interdisziplinäre Zusammenarbeit (zwischen der Person mit den Beschwerden, den therapeutischen Fachpersonen sowie den medizinischen Fachpersonen) kann der Diagnosestellungsprozess auf professioneller Ebene erfasst werden. Von den oben beschriebenen Definitionen ausgegangen, entsteht der Anspruch bezüglich des Diagnosestellungsprozesses, dass er verschiedene Ziele, Aufgaben und Funktionen erfüllen «muss». Laut Wittchen und Hoyer (2006) sind die Haupt- ziele dabei: Beschreibung des interessierenden Verhaltens, Erklärung, Vorhersage, Beeinflussung und Kontrolle, Intervention, Reduktion von Leiden, Behinderung und Verbesserung der Lebensqualität. Bei den Aufgaben in der Klinischen Psychologie geht es um quantitative und qualitative Beschreibungen der psychischen Störungen, Zuordnung zum Zwecke der Indikation bestimmter Klassen (im psychologischen und medizinischen Bereich in ICD-10, DSM-V, im Rehabilitationsbereich in ICF (Internationale Klassifikation der Funktionsfähigkeit, Behinderung und funktionalen Gesundheit), Entstehungsgeschichte bzw. die Bedingungen ihres Auftretens, ihren weiteren Verlauf bzw. die therapeutische Erfolgswahrscheinlichkeit vorhersagen, Information über den Behandlungserfolg liefern (Evaluation) (vgl. Krohne \& Hock, 2007; Bastine \& Tuschen, 1996) sowie Prognosestellung (vgl. Bastine, 1992). Dazu würde ich einen zusätzlichen Faktor einbringen, nämlich die «Fachidentität».

\section{Ein Mann sitzt im Warteraum.}

Als seine Therapeutin bitte ich ihn in mein Zimmer. Ich begrüsse ibn und stelle mich vor: "Ich bin Ihre Bezugstherapeutin, möchten Sie einen Tee oder ein Glas Wasser?»

Der Mann schaut mich mit grossen Augen an und fragt erstaunt: "Ich? Einen Tee vielleicht.»

Ich hole ihm einen Tee und lade ihn in mein Büro ein. In meinem Büro stehen zwei Stüble frei im Raum und ich biete dem Mann dort Platz an.

Dann bewegt er sich aufgrund seiner körperlichen Beschwerden schwerfällig durch den Raum, seine Körpersprache zeigt Unsicherheit. Höflich fragt er mich, welcher davon MEIN Stubl sei.

Ich erkläre ihm, dass er sich seinen Platz selbst wählen dürfe und setze mich ihm gegenüber.

Er kommentiert die Situation: «Hu! Eine Ärztin bringt mir einen Tee. Und ich darf meinen Sitzplatz wählen. Das ist doch ein Charakter! Ich füble mich menschlich und wie eine gesunde Person behandelt.» Er lächelt und ist emotional berührt.

Ich habe seine Akten vor dem Termin gelesen und weiss, welche Diagnosen im Vorgespräch erhoben worden sind. Ich antworte bewusst: "Ja, wissen Sie, Sie sind ein Mensch, so wie ich, und ich mag auch meinen Sitzplatz wäblen und einen feinen Tee bei der Arbeit trinken.»

Er lächelt und nimmt eine entspannte Sitzposition ein. Und so fangen wir mit unserer ersten Sitzung an.

Ich füble mich in diesem Prozess beworzugt wie eine «menschliche Teilnehmerin», und nicht wie ein "Jemand». Ein Jemand, der weiss, was der Klient braucht und entsprechend der eigenen Überzengung den Patienten instruiert. Als Folge würden meine Bedürfnisse den Prozess bestimmen und beeinflussen. Aus meiner therapeutischen Identität entscheide ich mich ganz bewusst dagegen, dieser Jemand zu sein.

Meiner Meinung nach kann die bewusste Entscheidung zum Diagnosestellungsprozess durch die jeweilige therapeutische Fachperson aufgrund ihrer eigenen therapeutischen Einstellung und persönlichen Erfahrungen und 
durch die Intersubjektivität und Reife ihrer Persönlichkeit beeinflusst werden.

Die Intersubjektivität kann im klassischen Sinn als «Stufe der psychischen Struktur, welche die Stadien von Subjektivität und Objektivität durchlaufen hat und diese dialektisch integriert» (Fischer, 2007, S. 594) definiert werden. Aus meiner Sicht handelt es sich einerseits um die psychische Ebene, in der die Fähigkeit zur empathischen und realen Perspektivenübernahme Grundbedingung ist, um sich und andere im momentanen Kontext zu verstehen, andererseits um die Verhaltens- und Kommunikationsgrundhaltung, die auf Authentizität, Dimensionalität und einem «lebenslangen Entwicklungsprozess» basiert, um eine gegenwärtige menschliche Beziehung anstatt «einer Beziehung zwischen einem kranken Individuum und einem gesunden Individuum» zu erzielen.

Die Grundkonzepte des klinischen diagnostischen Prozesses wurden bis heute mehrfach erforscht und oft erneuert oder neu definiert. Aufgrund des dimensionalen Modells von Stieglitz werden fünf Dimensionen voneinander unterschieden: unimodal vs. multimodal, dimensional vs. kategorial, störungsübergreifend vs. störungsbezogen, Statusdiagnostik vs. Verlaufsdiagnostik und theoriebezogen vs. nicht theoriebezogen (vgl. Stieglitz, 2008, S. 21).

Diese fünf Dimensionen behaupten den bestimmten Bedeutungsrahmen für die Diagnosestellung und beschreiben die Rahmenbedingungen für die daraus entstehenden Aufgaben und Ziele.

Dafür ziehe ich beispielsweise bezüglich der Depression aus der Sicht die Form der klassischen klinischen Diagnostik ICD-10 und auch Diagnostik aus Sicht des Inneren Teams in Erwägung. Die ICD beschreibt psychische Störungen von ibrer Phänomenologie her, also von ibren äusseren Escheinungsbildern, als Diagnosekriterien (zum Beispiel: depressive Verstimmung; deutlich vermindertes Interesse; deutlicher Gewichts-Appetitverlust; Schlaflosigkeit/vermehrter Schlaf; Psychomotorische Unruhel Verlangsamung; Müdigkeit/Energieverlust; Gefüble von Wertlosigkeit/Schuld; Konzentrations-und Entscheidungsprobleme; Tod, Suizidgedanken oder Handlungen etc.). Welche dieser Symptome muss man (PatientIn/KlientIn/ Person mit Leiden und Beschwerden) zeigen, damit ein Arztleine Ärztin oder TherapeutIn eine Depression diagnostizieren darf? Durch diese Kriterien können wir konkrete Kategorien vorausnehmen/erkennen und durch die Dimensionen von ICD-10 störungsbezogene Statusdiagnostik erstellen. Das Modell des Inneren Teams dagegen beschreibt die innere, persönliche, individuelle Dynamik. Diese führt zu den entsprechenden Symptomen und kann sehr unterschiedlich sein und ständig verändert werden. Bei Depression zeigt sich in der Regel, dass die Persönlichkeitsanteile («die Teammitglieder») mit starken Gefüblen abgespalten und weggesperrt worden sind. Ich würde sagen, die wachen Anteile, die verhindern, dass diese Mitglieder für andere sichtbar werden - und häufig auch, dass sie innerlich gespürt werden (vgl. Kumbier, 2013). So beginnt eine depressive Entwicklung, wenn ein sogenannter abgespaltener, innerlich «nicht zugehöriger» Persönlichkeitsanteil als Teammitglied (zum Beispiel verletztes Kind) geweckt wird. Spürbar ist, wie die wichtigen Begriffe bei dieser Diagnostik sind: dimensionale und nicht störungsspezifische, verlaufsorientierte Sicht und eine integrierte Denkweise sowie nicht vorhersehbare individuelle Aspekte oder Wenden. Diese Form von Diagnostik hat verschiedene Berührungspunkte mit der klassischen klinischen Diagnostik, vor allem, was die Verortung und Störungsstruktur angeht, zugleich jedoch steht der Ansatz quer zur klinischen Diagnostik mit dem ICD-10. Ich würde die folgende Fragestellung statt der oben genannten empfehlen: Was genau will ich gerade jetzt messen? Eher die Beschreibungskategorien oder die Funktionalität bzw. die aktuellen, individuellen Entwicklungsstände? Denn wir brauchen beide in der Psychotherapie und beide sind gleich unentbehrlich.

Als Fachperson «muss» man sich für etwas bewusst entscheiden, damit die weiteren Facharbeiten (Diagnosestellung und therapeutische Tätigkeit) vereinfacht werden können, etwa: Erfassung einzelner Facetten vs. Erfassung eines weiten Spektrums, Schweregraderfassung; Quantifizierung vs. Klassifikation; universelle und vergleichende Erfassung vs. spezielle (spezifische) konstruktnahe Ebene, Ist-Zustand (diagnostisch, prognostisch) vs. Prozess- und Veränderungserfassung, Therapieorientierung und Interventionsspezifizierung vs. allgemeine Einsetzbarkeit. Die Selektion zwischen den Aspekten und Dimensionen ist die eigene bewusste Wahl. So werden die diagnostischen Prozesse von der jeweiligen Strategie bewusst mitbestimmt.

Der Prozess der Diagnostik in der klinischen Psychologie, speziell der Psychotherapie, unterscheidet sich vom dem in anderen Diagnostikbereichen (zum Beispiel dem des Pädagogischen oder des rein Medizinischen) insofern, als er hier nicht primär «linear» angelegt ist, sondern «rekursiv». Die jeweils neu erhobenen diagnostischen «Daten» modifizieren oder ergänzen die bereits vorliegenden Daten und der oft schon mitlaufende Interventionsprozess produziert seinerseits ebenfalls neue «Daten» (vgl. Linster, 2010), laut Ganzach (1995) und Nietzel et al. (1994) «additiv» genannt.

«Daten» und «Informationen» sind kaum voneinander zu trennen. Bei beiden handelt es sich um einen Entdeckungs- und Aufdeckungsprozess, um eine «Konstruktion», entsprechend dem Diktum, dass die «endgültige» und/oder umfassende Diagnose erst am Ende einer Psychotherapie valide und reliabel vorliegt (vgl. Linster, 2010). Falls die Prozesshaftigkeit bevorzugt wird, kann sich ein sogenannter «offener Kanal» zwischen den Prozessbeteiligten entwickeln. Dadurch kann eine endlose Offenheit zur Authentizität, ein zeitlich nicht eingeschränkter Raum zur Entwicklung sowie eine permanent laufende «Kommunikation» entstehen, die eine auf Gegenwärtigkeit basierende Zusammenarbeit ermöglichen. An diesem Punkt wird das kategoriale Grundkonzept nicht kritisiert, sondern aus einer neuen Perspektive betrachtet, ähnlich wie ein Kettenglied in dem System, das in den 1960er Jahren im klinischen Bereich entstanden ist und durch die neuen 
Generationen auch weiterentwickelt wird. Durch eine integrierte Sichtweise, ohne Vor- und Beurteilung, ob das Beobachtete gut oder schlecht ist, kann ein therapeutisch integriertes dimensionales Konzept aufgebaut werden, in dem die Vorteile und Nachteile der verschiedenen Richtungen gleichgültig und gleichgewichtig auf eine Metaebene vereinigt werden können. Es kann als ein System für den klinischen Bereich angesehen werden, das anstelle von Erwartungen die eigene Grenzsetzung indiziert und anstelle einer Suche nach Mängeln die Unterschiede als schlicht gegeben akzeptiert werden. Diesen Prozess kann die Person mit Leiden und Beschwerden schon bei der Diagnosestellung erleben. Trotz ihrer Störung ist sie genau so ein Mensch wie die Hilfsperson und kann - wenn ihr dies entsprechend gespiegelt wird - die Hoffnung entwickeln, dass sie irgendwann mit ihrem Tempo und auf ihre Art und Weise "gesund genug» sein darf. Ein Ausgangspunkt für die Beteiligten, in dem die Aktualität die Hauptrolle spielt und nicht zukünftige Annahmen. In diesem Zusammenhang verstehe ich unter Begriff «gesund genug» ein «Ich bin ich, mitsamt meiner Stärken und Schwächen».

Ich finde, durch ein integriertes dimensionales Konzept kann der Prozess «Erkennen-Verstehen-Handeln-Evaluieren» eine ganzheitliche, respekt- und wertorientierende Grundeinstellung hervorrufen und sich dadurch eine Vielzahl an neuen Wegen zur Entstehung der Berufsidentität öffnen.

\section{Neuartige Möglichkeiten der Bedeutung von der Rollenannahme in dem Diagnosestellungsprozess}

In der Klinischen Psychologie, Rehabilitationspsychologie und Psychotherapie sind verschiedene Annahmen über den Menschen und Gesundheit bzw. Krankheit in Anwendung. Diese Annahmen werden von der jeweiligen Kultur und den gesellschaftlichen Lagen und Interessen entscheidend bestimmt (vgl. Röhrle, 2008).

Aus historischer Sicht war am Anfang (im klinischen Diagnosestellungsbereich/im Bereich der klinischen Diagnosestellung) der Aspekt «Menschen als Individuen zu verstehen, die sich voneinander unterschieden» verbreitet. Mit der Erscheinung des Eigenschaftmodells wurde es fachlich interessant, die interindividuellen Unterschiede anhand stabiler Merkmale zu typisieren (zum Beispiel im Trait-Modell). Durch das taxonomische Interesse wird eine Gelegenheit geboten zu erweiterten Überlegungen der Bedeutungsrahmen der Modellannahme - nicht nur bezüglich Individuen, sondern auch bezüglich Zuständen wie Krankheiten, psychische Störungen und Affekten (vgl. ebd.). Tendenz und Bedarf nach weiteren Betrachtungen war immer präsent im psychologischen Bereich und ist gültig auch für unsere heutige Situation. Die Wende ist auf einer sukzessiven Art und Weise seit den 1960er Jahren bis heute eingetreten: zu einer Milieuorientierung, zur «situationistischen Diagnsotik». Sie wurde bedingt durch: 1. Aus wissenschaftlicher Sicht definierten Kritiken (an der psychiatrischen Diagnostik und dem damit verbundenen medizinischen Krankheitsmodell),

2. die Einflüsse von Lerntheorien und Ökologischen Theorien,

3. das Verlieren der Personmerkmale zugunsten von Situationsmerkmalen an Gewicht bzw. «Interaktionismus»

4. Soziale Klimata,

5. die Erkenntnisse, dass durch Modifikation von Situationsmerkmalen individuelles Verhalten modifiziert werden kann

6. und die zunehmenden Interessen an modifikationsorientierter Diagnostik insgesamt.

In unserem 21. Jahrhundert werden die Ansprüche immer mehr an die energetischen und spirituellen Aspekte im diagnostischen und therapeutischen Bereich gestellt. Im Laufe der gesellschaftlichen Entwicklungen und damit veränderten Bedingungen entstehen neue diagnostische Aufgaben wie die Auswahl von qualifizierten Arbeitskräften, die Zuweisung bzw. Nichtzuweisung von Krankheitskategorien, um Arbeitskräfte zu erhalten oder wiederherzustellen (vgl. Röhrle, 2008; Linster, 2010). Durch Medienberichte, Online-Priorität und «aktuelle gesellschaftliche Erwartungen» entstehen die Veränderungen des gesellschaftlichen bzw. individuellen Umgangs mit psychischen Störungen, «Therapierbarkeit», "Gesundheit-Kranksein» und «Menschensein», was sich sowohl auf der individuellen Ebene als auch auf den anderen sozialen Ebenen auswirken kann. Dies kann ausserdem zu weiteren fachlichen Modifikationen führen, was auch wissenschaftlich nachgewiesen wurde:

«Aus Vergleichen von Mehrebenenanalysen ergab sich, dass die institutionelle Zugehörigkeit zu Therapierichtungen und/oder die subjektive Identifikation von TherapeutInnen mit bestimmten Therapiemethoden das tatsächliche Interventionsverhalten praktisch nicht vorhersagte. Vielmehr scheinen BehandlerInnen bis zu einem gewissen Grad einen persönlichen Stil entwickelt zu haben, der sie mehr oder weniger unterstützend, beratend, klärend, emotions-, deutungs-oder Therapieziel-orientiert arbeiten lässt (vgl. Tschuschke et al., 2016). Am deutlichsten sind es aber offensichtlich die PatientInnen selbst, deren Persönlichkeit und individuellen Bedürfnisse bestimmen, wie ihre TherapeutInnen die Sitzungen mit ihnen gestalten. Dies entspricht der von Lambert formulierten Forderung, wonach sich TherapeutInnen bemühen sollten, >matching techniques to client dispositions, personality traits and other diagnostic differences< (Lambert 2013, S. 206 und Norcross \& Wampold, 2011).

Der erstaunlichste Aspekt der modernen Psychotherapie ist die Anzahl konkurrierender Schulen und der Mangel an Übereinstimmung zwischen ihnen. Es bestehen grosse Differenzen über grundlegende Fragen: Was sind die Dimensionen der menschliche Psyche, und was sind ihre wichtigsten motivierenden Kräfte? Warum entstehen Symptome, und was bedeuten sie? Welche Themen, die die Klienten in die Therapie bringen, haben zentrale Bedeutung, und welche sind weniger relevant? Welche Techniken, Strategien oder diagnostische Mittel sollten zur Messung und Korrektur oder zur Verbesserung der emotionalen, psychosomatischen und 
der sozialen Situation der Klienten angewandt werden? Es gibt so viele Antworten auf die Fragen, wie es Schulen der Psychotherapie gibt» (Grof, 2015, L. 518).

Durch diesen Mangel an Übereinstimmung von Theorien und Praxis in der Psychotherapie kann ein sogenanntes Unbefriedigtsein entstehen. Unter diesen Umständen kann ein Klient mit einer emotionalen oder psychosomatischen Störung eine Psychotherapieschule quasi auswählen als werfe er eine Münze. Dann wird ein angehender Therapeut aufgrund der gelernten Theorien und Methoden damit umgehen, die Problematik zu überwinden. Vermutlich stellt er sich nicht die kritische Frage von Stanislav Grof: «Sagt seine Wahl mehr über die Persönlichkeit des Bewerbers aus als über den Wert der Schule?» Dadurch kann das Problem der sogenannten fachlichen Isolation hervorrufen werden: Die Dynamik wird durch die psychotherapeutischen Schulen auf einer bestimmten Ebene der Psyche korrekt und bestimmt beschrieben, ihnen fehlt aber das Verständnis der Phänomene aus anderen Ebenen des Bewussten. Sie versuchen dann meistens diese Erscheinungen in ihrem eigenen begrenzten konzeptionellen Rahmen zu interpretieren.

\begin{abstract}
«Ein wirksames und nützliches psychotherapeutisches System muss alle Ebenen der Psyche enerkennen und akzeptieren. Welche Inhalte in der Sitzung erforscht und verarbeitet werden, wird durch die Dynamik des Unbewussten des Klienten und seines eigenen psychologisches Prozesses bestimmt. Die TherapeutIn muss über einen genügend breiten konzeptuellen Rahmen verfügen, um seine KlientInnen auf allen Ebenen ihres Unbewussten begleiten zu können - biografisch, perinatal und/oder transpersonal - und ihre entsprechenden Erfahrungen zu unterstützen» (Grof, 2015, L. 485; Vaughan, 1993).
\end{abstract}

Wenn wir von dem integrierten psychotherapeurischen Konzept von Wolfgang Senf (2017) ausgehen würden, kann ein integriertes dimensionelles System dargestellt werden (siehe Abbildung 1). Beim ersten Treffen mit einer Person mit Leiden und Beschwerden treten wir durch den Diagnosestellungsprozess in den psychotherapeutischen Prozess «ein». Automatisch fängt die Kommunikation zwischen den Prozessbeteiligten in der Phase «Erkennen» an. Wie ich schon früher erwähnt habe, ist die Diagnosestellung

Abb. 1:

Dimensionen Integrativer Psychotherapie (Senf, 2017). schon ein wichtiger Schritt in der Psychotherapie. Seine Auswirkung kann auch sehr prägend sein.

Anhand Abbildung 1 kann eine mögliche integrierte Struktur erfasst werden, wodurch ein universelles jedoch individuelles therapeutisches Modell als Bedeutungsmöglichkeit darlegt werden kann.

An dieser Stelle möchte ich die Rolle der subjektiven bzw. interaktionellen Faktoren abwägen, die ein integriertes Bild von der therapeutischen Einstellung zeigen können, deren Auswirkungen den ganzen therapeutischen Prozess beeinflussen werden. Dabei halte ich die Gegenwärtigkeit und die Autenthizität, die diese oben erwähnte integrierte und dimensionelle Sichtweise «fundieren» können, für wichtig.

Mit Absicht bezeichne ich in diesem Beitrag eine "Person mit momentanen Leiden und Beschwerden", die zur Therapie kommt und zur Diagnosestellung geht, nicht als «PatientIn» oder «KlientIn». Ich mache einen Versuch, einen neuen Terminus dafür einzuführen, eine unpolarisierte Grundposition zu vermeiden. Aus meiner Sicht fängt schon die therapeutische Wirkung mit der Benennung und «Anrede» an.

Auf Grundlage der transaktionalistischen Sicht kann eine «Ich bin ok, du bist ok» («dein Leiden ist aktuell») Botschaft direkt und indirekte Weise vorausgesetzt und kommuniziert werden, sodass es möglich wird, eine balancierte und auf Menschlichkeit basierende soziale Position von Anfang an herzustellen, die rasch und unbewusst ein heilendes Muster hervorruft und dadurch einen heilenden Input gibt, entsprechend zu einem «Reiz» werden kann. Es ist nicht einfach und selbstverständlich, sondern abhängig von der Reife der Persönlichkeit der jeweiligen TherapeutIn - vom «Facilitator», wie Stanislaw Grof es ausdrückte, bzw. von Yaloms «Wegbegleiter» (Yalom, 2013, S. 318). Aus meiner Sicht ist er ein Fachbegleiter, Unterstützer und

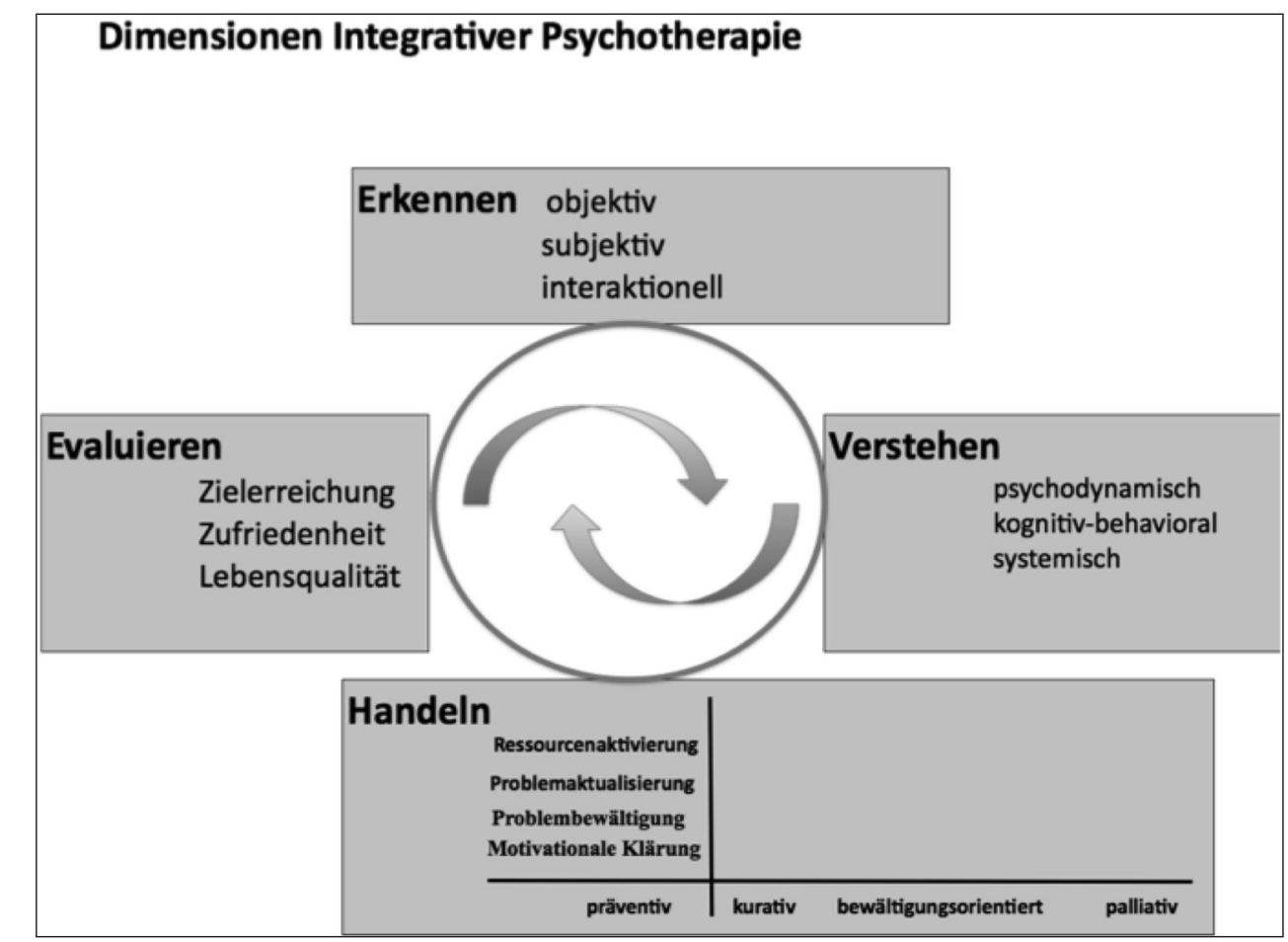


"Gleichmensch» ${ }^{1}$, mit Stärken und Schwächen, der den therapeutischen Prozess für eine bestimmte Zeit begleitet und sich darauf konzentriert, mit der ihm gegenübersitzenden Person mit Leiden und Beschwerden wahrhaftig seelisch und körperlich zusammenzuarbeiten. Zur Verfügung steht ihm die Fähigkeit und Bereitschaft, die Maske der Autorität wegzunehmen und nicht in einer Rolle zu erscheinen. Es ist nämlich kontraproduktiv, wenn die TherapeutIn für die Person mit Leiden und Beschwerden undurchsichtig und im Verborgen bleibt. Alles spricht dafür, sich der Person mit Leiden und Beschwerden zu enthüllen, und nichts für einen Gewinn durch Heimlichkeit. Wenn ich als therapeutische Begleiterin dieses Thema anspreche, stosse ich trotzdem oft auf erhebliches Unbehagen, das zum Teil von der Ungenauigkeit des Begriffs «Selbstoffenbarung» herrührt. Laut Yalom (2013) ist die therapeutische Selbstoffenbarung, keine einzelne Verhaltensweise, sondern eher ein sogenanntes Bündel von Verhaltensweisen, von denen einige unweigerlich die Therapie erleichtern und andere problematisch und potenziell kontraproduktiv sind.

Das Wichtigste, was wir TherapeutInnen einem zu uns kommenden Menschen mit Leiden und Beschwerden geben können, ist eine authentische, heilsame Beziehung, die es ihm ermöglicht, nach und nach zu gesunden. Wir machen uns etwas vor, wenn wir glauben, dass irgendeine bestimmte Aktion, sei es Interpretation, Suggestion, Relabelling oder Bestärkung der Heilfaktor schlechthin ist. Für die Personen mit Leiden und Beschwerden ist es wichtig, dass eine für ihn bedeutsame Person ihn als bedeutsam erachtet.

Seit ich mich für diesen Weg enschieden habe, will ich authentisch in der Psychotherapie reagieren und nicht mehr "gewinnen» oder «besser wissen", will nicht mehr die Kontrolle über einen anderen Menschen (zum Beispiel über Personen mit Leiden oder Beschwerden) oder über eine Situation mir aneignen, weil ich weiss, dass es weder richtig noch fruchbar ist, jemand überzengen oder mich verteidigen $z u$ wollen. Ich versuche, einfach ich $z u$ sein, auch wenn ich einen Fehler mache (zum Beispiel ein Formular vergesse in die Sitzung mitzubringen etc.), $z u$ anderen Menschen in Beziehung zu treten und eine direkte, wabre, menschliche, gegenwärtige Verbindung $z u$ ihnen herzustellen. Mein therapeutisches Handeln gründet in einer Position der Fürsorge, Achtsamkeit und des «Daseins» für mich bzw. für uns selbst und für die Person mit Leiden oder Beschwerden, mit einem Bewusstsein für den momentanen Kontext. In meiner klinischen Arbeit bemerke ich seitdem eine sehr wesentliche positive Auswirkung, wie ich «mit meinen Fehlern oder Unsicherheiten» während der Sitzung umgehe und teilweise kommuniziere. Das macht mich menschlich und die anderen auch. Sobald ich bei Person mit Leiden zurückfrage: «Dürfen Sie auch Fehler machen?» und ich mit Gegenwärtigkeit auf ibre Antworten reagiere ( $A h a$, wir dürfen auch alle, weil wir Menschen sind.») oder/und

1 Das heißt: zwei Menschen sprechen auf Augenhöhe. provokant, wenn die Person mit Leiden «Nein» antwortet: «Ach schade, ich finde einen Fehler zu machen, kann ganz hilfreich und menschlich sein.» Die Personen spüren und äussern, dass sie danach ein bisschen erleichtert sind.

Virginai Satir forderte die TherapeutInnen dazu auf, auf der Ebene Kongruenz, der allgemeinen Gleichwertigkeit der Menschen Kontakt zu ihren KlientInnen aufzunehmen. Dies wird eine Befähigung (empowerment) beider Seiten implizieren, und es intensiviert bzw. legalisiert auch auf beiden Seiten das Gefühl innerer Stärke. Gleichzeitig wird auf die Bedeutung des beispielhaften Vorlebens (modeling) von Kongruenz hingewiesen, was die Wachstumsmöglichkeiten der Person mit Leiden und Beschwerden erheblich steigert (vgl. Satir et al., 2011). Kongrunz bezeichnet sowohl einen Seinzustand als auch eine Qualität des Kommunizierens. Es betrifft verschiedene Komponenten sowie verhaltens- und kommunikatiosnbezogene, kognitive-, emotionelle und konative Faktoren.

Zum Erreichen der Kongruenz können die folgenden wichtigsten verhaltens-, und kommunikatiosnbezogene Schritte benannt werden:

«1. Sei dir dessen bewusst und akzeptiere, dass du Wahlmöglichkeiten hinsichtlich deiner Art zu antworten hast.

2. Bleibe auf allen Ebenen (Erwartungen, Wahrnehmungen und Gefühlen) mit all dem in Kontakt, was in der Gegenwart in dir vor sich geht.

3. Stehe für das ein, was von dir kommt, seien es Worte, Körperbotschaften oder Handlungen.

4. Kommuniziere und antworte mit Ich-Botschaften.

5. Vermittle klar, dass du für das, was du sagst oder tust, einstehst.

6. Teile in beschreibender - statt in urteilender Sprache mit, was in dir vor sich geht sowie auch was du die andere Person sagen und tun siehst und hörst.

7. Schenke der psychischen Distanz und Nähe Beachtung, für die du dich entscheidest, wenn du zu jemandem in Kontakt trittst. Richte es so ein, dass du dich, in Augenhöhe und in einer dir angenehmen Nähe zu jener Person befindest» (Satir et al., 2011, S. 100).

Beim Einüben von Kongruenz können die folgenden kognitiven Komponenten für wichtig gehalten werden: Überzeugungen von menschlichen Fähigkeiten, die Grundeinstellung der eigenen Position, die Grundeinstellung von Aufmerksamkeitniveau und Wahrhaftigkeit (vgl. ebd.), Sinnbegreifungsvermögen, Bereitschaft für «Offensein für alles» sowie dimensionell definierte Selbstdisziplin (vgl. Krasznai, 2011).

Durch die konativen Komponenten können das Wertesystem, die Einstellung von Verantwortungsannahme und «Konsequentsein» definiert und vorausgesetzt werden.

Aufgrund des Persönlichkeitsmodells von Éva Berentés kann diediesbezügliche Persönlichkeitsstruktur, als die Struktur der Reife Persönlichkeit ausführlich und einzigartig erfasst werden:

«I. Kognitive Eigenschaften der reifen Persönlichkeit I.1. reale Selbst-und Wirklichkeitswahrnehmung = wirklichkeitsgetreue/wahre Wahrnehmung 
$>$ Fähigkeit der Unterscheidung zwischen Meinungen und Tatsachen

$>$ reale-, positive Selbststrukturen

$>$ reifes Realitäts-, Massbewusstsein

I.2. Innovation (Befähigung zu Neuem)

I.3. Integrität als Lebensphilosophie, spirituelle Reife

II. Interpersonelle Eigenschaften der reifen Persönlichkeit

II.1. in den Beziehungen: annehmend

$>$ beurteilungslos

$>$ Respekt für die Grenze von anderen

II.2. Bedürfnis nach gegenseitigen Beziehungen

II.3. Emotionale Intelligenz

III. Konative Eigenschaften der reifen Persönlichkeit

III.1. wirksame Lebensführung

III.2. in Balance zu sein bzgl. Lebensführung, Denken, Handlung

III.3. Stabilität in den Gewissensfunktionen

III.4. Assertivität» (Berentés, 2012, S. 205)

All das macht uns auf Selbsterfahrungen noch mehr aufmerksam. Dadurch kann eine reale und adäquate, wahre und individuelle Wahrnehmung sowie eine authentische therapeutische Zuwendung entwickelt werden.

Durch dieses bewusste Rollenannehmen kann die persönliche therapeutische Rolle und daraus folgende therapeutische Zielsetzung entstehen, die in den ganzen Prozess heilsam einwirken und diesen dadurch kontinuerlich ändern und weiterentwickeln kann. Dies ist eine gewaltige herausfordernde und niemals endende Aufgabe: unser Selbst zu erkennen (vgl. Itten, 2016). Was kann eine grenzenlose Freiheit, die Verantwortung und eine ganz neu interpretierte Hierarchie ohne soziale Distanz bieten?

Aktuell wird in Psychotherapiestunden zu stark vermittelt, wie wichtig eine Diagnose ist. Meistens fordern die Standard-Krankenversicherungen bzw. Krankentagegeld-Versicherungen bewusst, dass die ÄrztInnen und TherapeutInnen rasch eine präzise, wissentschaftlich nachgewiesene und codifizierte Diagnose stellen, um dann mit einer kurzen, Leitlinien entsprechenden, störungsspezifischen Therapie zu beginnen, die der jeweiligen Diagnose entspricht. So kann eine mögliche Prognose und ein zugehöriger Behandlungsplan aufgebaut werden. Das klingt professionell, logisch und effizient. Mit der Realität hat es nicht unbedingt viel zu tun. Es stellt nämlich den illusorischen Versuch dar, wissenschaftliche Genauigkeit bzw. «Berechenbarkeit» zu erwirken, wo sie weder möglich noch wünschenswert ist. Wir verlieren nämlich unsere Spontaneität und Offenheit für nicht vorhersagbare Reaktionen derjenigen Menschen, die zu uns kommen, um Hilfe zu erhalten.

Ich finde, die Psychotherapie ist ein sich langsam entfaltender, dynamischer, konstruktiver, komplexer «Energieprozess», der aus unserer Zusammenarbeit entsteht und in dessen Verlauf die TherapeutIn versucht, die PatientIn so gut wie möglich im Hier und Jetzt kennenzulernen. Falls wir eine Diagnose gestellt haben, können wir dazu neigen, diejenigen Seiten der PatientInnen, die nicht $z u$ der jeweiligen Diagnose passen, selektiv auszugrenzen und entsprechend übermässig aufmerksam zu sein für subtile Eigenarten, die unsere sogenannte Anfangsdiagnose zu bestätigen scheinen (vgl. Yalom, 2010). Jemanden als Borderline-PatientIn einzustufen, kann ganz schnell dazu beitragen, dass genau diese Züge stimuliert und verfestigt werden, weil wir die störungsspezifischen Merkmale kennen, wodurch wir unser Blickfeld verengen sowie unsere Fähigkeit mindern, den anderen als einen "Menschen» wabrzunehmen.

Eine Diagnose ist fraglos entscheidend beim Erwägen der therapeutischen Behandlung von klinischen Störungen mit biologischem Substrat, kann sich jedoch ganz kontraproduktiv ohne Bewusstsein der gegenwärtigen Wabrnehmung auswirken. Für mich geht es bei der Diagnosestellung ebenso wie in der Psychotherapie um die Persönlichkeitsentwicklung: «sich selbst weiter zu schärfen und reifen».

An dieser Stelle muss ich auch die Rolle und Wichtigkeit der Bedeutung anderer Personen mit (vergleichbaren) Leiden erwähnen. Die Personen mit Leiden und Beschwerden lernen nämlich voneinander nicht nur, wie sie mit Symptomen umgehen können, sondern auch, ob sie «richtige Borderliner» oder eher der «impulsive Typ» sind. Sie identifizieren sich nicht nur mit den Diagnosen oder mit den darauf folgenden Therapieerfolgen, sondern auch mit den schlechten und positiven Erfahrungen und Verhaltensweisen der anderen, nicht wenige lernen auf diesem Weg ihr Scheitern. Für mich als Mensch und auch als Psychologin ist es wichtig, den ratsuchenden Personen bei der Wahrnehmung von individuellen Unterschieden zu helfen, und ihnen meine Unterstützung anzubieten, damit sie mit diesen Unterschieden ohne Beurteilung umgehen sollen. Und dies gilt auch für mich. Denn genau dies heisst für mich Akzeptanz und authentische therapeutische Begleitung, weil ich weiss aus meiner therapeutische Selbsterfahrung, dass dahinter unglaublich viel Kraft, Energie, Arbeit, «Schweiss» und «Menschlichkeit» steckt.

\section{Fazit}

Heutzutage werden mindestens 400 verschiedene therapeutische Vorgehensweisen im klinischen Bereich angewendet (vgl. Garfield \& Bergin, 1994; Karasu, 1992) und die diagnostischen Möglichkeiten sowie das therapeutische «Methodeninventar» werden durch weitere Forschungen und durch neue Aspekte ergänzt und erweitert. Dies führt dazu, dass zwar eine unglaubliche Vielfalt und dadurch keine prinzipielle Einseitigkeit im Behandlungsfeld existiert, jedoch auch das Risiko zur Isolation der selbst ausgewählten therapeutischen Richtung/Sichtweise birgt. Zentrale Fragen im Umgang mit dieser Situation sind: «Wo positioniere ich mich im therapeutischen Prozess?»; «Wie definiere ich mich und wie definiere ich meine Verantwortung in diesem Prozess?» und «Was ist mein Ziel für den therapeutischen Prozess seit der ersten Begegnung mit der Person mit Leiden/Beschwerden?». Zwar geht es aktuell um das Thema klinische Diagnosestellung, aber dies ist schon ein wichtiger erster Schritt 
zur Therapie. Diese erste Begegnung kann sich günstig und/oder ungünstig auf den ganzen Therapieverlauf auswirken. In diesem ersten Schritt geht es darum, den «Grundstein» zu legen, als «Begleitperson, Hilfsperson oder vielleicht Vorbild», sowie man sich (mit-)bestimmt/ charakterisiert. Zudem wird dabei - durch Intersubjektivität -die eigene therapeutische Identität bewusst und die Reife der Persönlichkeit entwickelt.

Der individuell definierte Bedeutungsrahmen der Diagnosestellung und die Rolle von TherapeutInnen in der Psychotherapie entsteht schon über eine sich selbst definierte Ausgangsposition, die den weiteren Prozess beeinflusst. Wird ein Individuum mit Beschwerden als krank (Patient oder Patientin) angeschaut oder sogar als gehandikapt behandelt, hat das zur Folge, dass sie sich an diese Rolle und den damit einhergehenden Erwartungen bewusst bzw. unbewusst anpasst. Unbewusst werden diese Personen sich in die Rolle «minderwertig, weniger als du» hineinbegeben (vgl. Comer, 2005). Durch die therapeutische Kommunikation bestätigt, wird sich auf diesem Muster die von den TherapeutInnen nicht gewollte Vorstellung «Ich bin nicht ok, du bist ok»-Lebenseinstellung aufbauen (vgl. Berne, 2010). Dadurch kann schnell eine hierarchische Position in der therapeutischen Beziehung entstehen, mit der den Kriterien der Gegenseitigkeit geschadet wird. Virginia Satir ermahnt die Psychotherapeuten und Psychotherapeutinnen daher wie folgt: «Du bist deinen Klienten und Klientinnen snur ein bisschen voraus. Im Wissen, dass Veränderung möglich ist und der Wunsch da ist, Veränderungen vorzunehmen, dies sind zwei ersten grossen Schritte» (Satir et al., 2015, S. 105).

Abschliessend bleibt meine Fragestellung ohne eine konkrete Antwort. Es regt jedoch dazu an, eigene Überlegungen anzustellen: Wäre es irgendwann möglich mittels integrierender Art und Weise sowohl an der eigenen Selbstentwicklung wie auch in der psychotherapeutischen Situation zu arbeiten und beide Seiten der Therapie zusammen bewusst zum Ziel zu machen? Schon während des Diagnosestellungsprozesses? Und zwar unabhängig davon, welchen Status man hat: Die Person mit Leiden und Beschwerden und die Person mit Fachwissen über körperliche/organische sowie psychische Beschwerden agieren gleichwertig. Bleibt das eine Utopie des 21. Jahrhunderts oder kann verwirklicht werden, dass die Eigenverantwortung durch einen offenen, auf Gegenwertigkeit basierenden dynamischen Prozess definiert wird, anstatt durch eine polarisierte Sichtweise, die gesetzmässig eine statusorientierte und machtorientierte Selbstbestimmung zur Folge hat, die PatientInnen in eine Hierarchie drängt? $\mathrm{Ob}$ das ärztliche oder therapeutische "Scheinbild» in der arbeitsbezogenen Position eher auf Menschlichkeit basiert und sich die Integrität der Betonung von Ausschliesslichkeit fortzieht, das kann jeder/jede für sich selbst entschieden. Alle haben Wahl und das Potenzial zur Entscheidung!

\section{Literatur}

Bakos, F. (2013). Idegen szavak szótára. Budapest: Akadémia Kiadó. Bastine, R. \& Tuschen, B. (1996). Klinisch-psychologische Diagnostik. In A. Ehlers \& K. Hahlweg (Hrsg.), Enzyklopädie der Psychologie: Klinische Psychologie: Band 1. Grundlagen der Klinischen Psychologie (S. 195-268). Göttingen: Hogrefe.

Bastine, R. (1992). Klinische Psychologie (Band 2). Klinische Psychodiagnostik, Prävention, Gesundheitspsychologie, Psychotherapie, pschosoziale Intervention. Stuttgart/Berlin/Köln: Kohlhammer. http://digi.ub.uni-heidelberg.de/diglit/bastine1992bd2 (19.02.2018).

Barwinski, R. (2017). Intersubjektivität und Selbstreflexion. Psychotherapie-Wissenschaft, 7(1), 23-31.

Berne, E. (2010). Emberi játszmák. Budapest: Háttér Kiadó.

Bengel, J. \& Helmes, A. (2005). Rehabilitation. In M. Perrez \& U. Baumann (Hrsg.), Lehrbuch Klinische Psychologie - Psychotherapie (3., vollständig überarbeitete Auflage, S. 430-553). Bern: Huber.

Berentés, É. (2012). Az érett személyiség. Az emberi siker, a hatékonyság és a boldogulás személyiségháttere. Budapest: Pro Personal Kiadó.

Comer, R.J. (2005). A lélek betegségei. Pszichopatológia. Budapest: Osiris Kiadó.

Fischer, G. (2007). Kausale Psychotherapie. Kröning: Asanger.

Ganzach, Y. (1995). Nonlinear models of clinical judgment: Meehl's data revisited. Psychol. Bull., 118(3), 422-429.

Garfield, S.L. \& Bergin, A.E. (1994). Introduction and historical overview. In A.E. Bergin \& Garfield (Hrsg.), Handbook of psychotherapy and behavior change. New York: Wiley.

Geller, J.G., Norcross, J.C. \& Orlinsky, D.E. (2005). The Psychotherapist's Own Psychotherapy: Patient an Clinician Perspectives. Oxford, Univertisty Press.

Grof, S. (2015). Revision der Psychologie. Das Erbe eines halben Jahrhunderts Bewussteinsforschung. Solothurn: Nachtsschattenverlag AG.

Grof, S. (2008). A jöv pszichológiàja. Budapest: Pilis-Print Kiadó.

Harris, Th. A. (2011). Ich bin o.k. Du bist o.k. Wie wir uns selbst besser verstehen und unsere Einstellung zu anderen verändern können - Eine Einführung in die Transaktionsanalyse. Hamburg: Rowohlt Verlag.

Itten, Th. (2014). Grössenwahn. Ursachen und Folgen der Selbstüberschätzung. Zürich: Orell Füssli Verlag AG.

Itten, Th. \& Roberts, R. (2016). Politik der Erfahrung. Kritische Überlegungen zur Entwicklung von Psychologie und Psychotherapie. Gießen: Psychosozial-Verlag.

Jäger, R. S. \& Petermann, F. (Hrsg.). (1999). Psychologische Diagnostik. Ein Lehrbuch (4. Auflage). Weinheim: Beltz, PVU.

Karasu, T. B. (1992). The wors of times, the best of times. J. Psychother. Prac. Res., 1, 2-15.

Kumbier, D. (2013). Das innere Team in der Psychotherapie. Methoden- und Praxisbuch. Stuttgart: Klett-Cotta.

Krohne, H.W. \& Hock, M. (2007). Psychologische Diagnostik. Grundlage und Anwendungsfelder. Stuttgart: Kohlhammer GmbH.

Krasznai, A. (2011). Diplomarbeit: Die psychologischen Aspekte der personenzentrischen Rollenannahme als Lehrer: Die vergleichende Untersuchung der disziplinierten Persönlichkeit formenden Faktoren im Zusammenhang der Ideologie der Erzieher und Erzieherinnen. Budapest: ELTE.

Kriz, J. (2014). Grundkonzepte der Psychotherapie. Weinheim/Basel: Belz Verlag.

Lambert, M.J. (2013). The efficacy and effectiveness of psychotherapy. In M.J. Lambert (Hrsg.), Bergin and Garfield's handbook of psychotherapy and behavior change (6. Aufl., S. 169-218). Hoboken, NJ: John Wiley \& Sons.

Linster, H.W. (2010). Psychologische Diagnostik in der Klinischen Psychologie, rehabilitationspsychologie und Psychotherapie. Einführung und Ueberblick. Online Handout. https://www.psychologie.unifreiburg.de/studium.lehre/bachelor.of.science/skripte/frueheresem.html/lpm/lpmlinster/klindiagno (06.10.2017).

Nietzel, M. T., Bernsteil, D. A. \& Milich, R. (1994). Introduction to clinical psychology (4. Auflage). Englewood Cliffs, NJ: Prentice Hall. 
Norcross, J. C. \& Wampold, B. R. (2011). What works for whom: Tailoring psychotherapy to the person. Journal of Clinical Psychology, 67(2), 127-132.

Orlinsky, D.E. \& Ronnestad, M.H. (2005). Therapeutic work and professional development:Main findings and practical implications. Psychotherapy Bulletin, 40, 20-37.

Röhrle, B. (2008). Aufgaben und Hintergründe. In B. Röhrle, F. Caspar \& P.F. Schlottke (Hrsg.), Lehrbuch der klinisch-psychologischen Diagnostik (S. 13-29). Stuttgart: Kohlhammer.

Satir, V., Banmenn, J., Gerber, J. \& Gomori, M. (2015). A Satir-modell. A családterápia és ami azon túl van. Budapest: Ursus Libris.

Satir, V., Banmenn, J., Gerber, J. \& Gomori, M. (2011). Das Satir-Modell. Familientherapie und ihre Erweiterung. Paderborn: Junfermann.

Sedlak, F. \& Gerber G. (1976). Beziehungs als Therapie. Therapie als Beziehung. Michael Balints Beitrag zur heilenden Begegnung. http:// www.unsere-jugend.de/pdf/volltext012572.pdf (01.11.2017).

Senf, W. (2017). Psychotherapeutische Beziehung. Alles gleich doch verschieden? Perspektiven der psychotherapeutischen Schulen. Online Handout. https:/www.pukzh.ch/default/assets/file/handout prof._w._senf.pdf (12.10.2017).

Schmale-Rieder, A. (2016). Der bewusste Lebensplan. Das Skript in der Transaktionsanalyse. Thyische Muster und therapeutische Strategien. München: Kösel-Verlag.

Stamateas, B. (2015). Toxische Gefüble. Wie wir emotionale Verletzungen heilen können und inneren Frieden finden. München: dtv.

Stieglitz, R.-D. (2008). Klinische Diagnostik. Klinische Diagnostik und Evaluation, 1, 19-40.

Strotzka, H. (1975). Psychotherapie: Grundlagen, Verfahren Indikationen. München: Urban \& Schwarzenberg.

Tschuschke, V., von Wyl, A., Koemeda-Lutz, M., Crameri, A., Schlegel, M. \& Schultess, P. (2016). Die Bedeutung der psychotherapeutischen Schulen heute. Geschichte und Ausblick anhand einer empirischen Untersuchung. Psychotherapeut, 61(1), 54-65.

Vaughan, F. (1993). Healing and Wholeness: Transpersonal Psy Chotherapy. In R. Walsh \& F. Vaughan (Hrsg.), Paths Beyond Ego: The Transpersonal Vision (S. 160-165). Los Angeles, CA: J. P. Tarcher.

Wittchen, H-U. \& J. Hoyer (Hrsg.). (2006). Klinische Psychologie und Psychotherapie. Heidelberg: Springer.

Wyl, von A, Tschuschke, V., Crameri, A., Koemeda-Lutz, M. \& Schultess, P. (2016). Was wirkt in der Psychotherapie? Ergebnisse der Praxisstudie ambulante Psychotherapie zu 10 unterschiedlichen Verfahren. Gießen: Psychosozial-Verlag.

Yalom, D. Y. (2008). Ein menschliches Herz. München: btb Verlag in der Verlagsgruppe Random House GmbH.

Yalom, D. Y. (2010). Der Panama-Hut oder Was einen guten Therapeuten ausmacht. München: Goldmann Verlag.

Yalom, D. Y. (2013). A magyar macska àtka. Pszichoteràpiàs mesék. Budapest: Park Könyvkiadó.

Yalom, D. Y. (2015). Denn alles ist vergänglich. Geschichten aus der Psychotherapie. München: btb Verlag in der Verlagsgruppe Random House GmbH.

\section{A reflection on an integrated perspective in psychotherapy}

What determines the opinion of the psychotherapist during the psychotherapeutic process?

The role of diagnostics in the psychotherapeutic process is questionable in various ways: On the one hand, clinical diagnostics demonstrate a commitment to a pathology- and deficit-oriented medical model from which the indications for therapy are developed. The existence of various forms of therapy, on the other hand, tie the diagnosis of mental disorders to line-specific phenomena for which the medical model has only limited applicability. The question may be provocative but also forward-looking. Depending on the individual level of specialist interest or position one can follow the explanations.

My contribution attempts to address some aspects along with the resulting questions and problems, as well as the advantages of medical cooperation in the context of clinical diagnosis and to create an individual space for it in psychotherapy through the use of «disciplinary identity».

Keywords: Clinical Diagnostics, Diversity, Authenticity, Models, Role, Psychotherapy, Disciplinary Identity

\section{Una riflessione da un punto di vista integrato nell'ambito della psicoterapia}

Da cosa dipende la risposta dello/a psicoterapeuta nel corso del processo psicoterapeutico?

Nel processo psicoterapeutico il ruolo della diagnostica risulta dubbio in molti rispetti: da una parte la diagnostica si trova obbligata a un modello medico orientato alla patologia e alla carenza, a partire dal quale vengono impostate le indicazioni per una terapia. D'altro lato a causa dei diversi indirizzi terapeutici la diagnostica dei disturbi psichici si rivolge a campi fenomenici specifici in cui viene necessariamente utilizzato il modello medico. La questione può essere provocante ma anche orientata al futuro. Per ogni livello individuale di interesse specialistico o in modo corrispondente all'impostazione possono seguire le argomentazioni.

Il mio contributo tenta di tematizzare alcuni aspetti e le domande e i problemi da essi derivanti nonché i vantaggi riguardo alla collaborazione medica nella diagnosi clinica e dare loro una posizione individuale nella psicoterapia grazie all'«identità specializzata».

Parole chiave: diagnostica clinica-psicologica, diversità, autenticità, modelli, ruolo, psicoterapia, identità specializzata

\section{Die Autorin}

Angéla Szalontainé Krasznai, MSc, Studium an der Universität ELTE Budapest (Ungarn), ist delegierte Psychotherapeutin im ambulaten Rahmen im psychosomatischen Bereich. Sie ist Mitglied in folgenden Organisationen und Verbänden: FSP (Föderation der Schweizer Psychologinnen und Psychologen), SSCP (Swiss Society for Coaching Psychology), SAPPM (Schweizerische Akademie für Psychosomatische und Psychosoziale Medizin), ICF (International Coach Federation) und aussenordentliche Mitglied der Systemis. ch (Schweizerische Vereinigung für systemische Therapie und Beratung). Ihre Forschungsschwerpunkte sind die Analyse der Persönlichkeitsentwicklung sowie Förderung der Motivationsbasis bzw. Disziplin.

\section{Kontakt}

Angéla Szalontainé Krasznai, Psychologin FSP

Waldeggstrasse 1

CH-9500 Wil

Schweiz

E-Mail: smileka@gmail.com 